PROCEEDINGS OF THE

AMERICAN MATHEMATICAL SOCIETY

Volume 125, Number 7, July 1997, Pages 2013-2020

S 0002-9939(97)03784-2

\title{
WEIGHTED WEAK-TYPE INEQUALITIES FOR THE MAXIMAL FUNCTION OF NONNEGATIVE INTEGRAL TRANSFORMS OVER APPROACH REGIONS
}

\author{
SHIYING ZHAO
}

(Communicated by J. Marshall Ash)

\begin{abstract}
The relation between approach regions and singularities of nonnegative kernels $K_{t}(x, y)$ is studied, where $t \in(0, \infty), x, y \in X$, and $X$ is a homogeneous space. For $1 \leq p<q<\infty$, a sufficient condition on approach regions $\Omega_{a}(a \in X)$ is given so that the maximal function

$$
\sup _{(x, t) \in \Omega_{a}} \int_{X} K_{t}(x, y) f(y) d \sigma(y)
$$

is weak-type $(p, q)$ with respect to a pair of measures $\sigma$ and $\omega$. It is shown that this condition is also necessary for operators of potential type in the sense of Sawyer and Wheedon (Amer. J. Math. 114 (1992), 813-874).
\end{abstract}

\section{INTRODUCTION}

In [6], A. Nagel and E. Stein improved the classical theorem of Fatou on nontangential limits of harmonic functions defined on $\mathbb{R}_{+}^{n+1}$ to include limits within regions which allow sequential approach with any degree of tangency to the boundary, $\mathbb{R}^{n}$. Their proof is based on a remarkable result of a necessary and sufficient condition on the approach regions so that the associated maximal function of Hardy-Littlewood type is weak type $(1,1)$. The condition gives a clear picture of the relation between the size of cross-sectional measure of approach regions at height $r$ and the "singularity", $r^{-n}$, of the maximal operator of Hardy-Littlewood type. Following this important work, a series of papers has been devoted to maximal functions of Hardy-Littlewood type for associated approach regions (see, e.g. [1], [9], [5], and [7]). In this paper, we show that a similar relation holds for general integral transforms with nonnegative kernels in the upper half-space of a homogeneous space. We give a sufficient condition, which turns out to be necessary for operators of potential type in the sense of [8], on approach regions so that weak-type $(p, q)$ $(1 \leq p<q<\infty)$ inequalities (see (1.6) below) hold for the maximal function over the approach regions with respect to a pair of Borel measures $\sigma$ and $\omega$ on $X$. Our condition and proof are motivated by a recent paper [3], where some general weak-type inequalities for integral transforms with nonnegative kernels are studied.

Received by the editors April 13, 1994 and, in revised form, January 19, 1996.

1991 Mathematics Subject Classification. Primary 42B20, 42 B25.

Key words and phrases. Weak type inequalities, weights, operators of potential type, maximal functions, approach regions. 
A homogeneous space $(X, d, \mu)$ is a set $X$ together with a quasi-metric $d$ and a doubling measure $\mu$. By the quasi-metric $d$ we mean a mapping $d: X \times X \rightarrow[0, \infty)$ which satisfies the same conditions as a metric, except the triangle inequality is weakened to

$$
d(x, y) \leq \kappa(d(x, z)+d(z, y)) \quad \text { for all } x, y, z \in X
$$

where $\kappa \geq 1$ is a constant which is independent of $x, y$, and $z$. Without loss of generality, we may assume that all balls $B(x, r)=\{y \in X: d(x, y)<r\}$ are open (see [4]). We also use the convention that $B(x, 0)=\varnothing$. By the doubling measure $\mu$ we mean a nonnegative measure on the Borel subsets of $X$ so that $|2 B|_{\mu} \leq C_{\mu}|B|_{\mu}$ for all balls $B \subset X$, where $|B|_{\mu}$ denotes the $\mu$-measure of the ball $B$, and $C_{\mu}$ is a constant which depends only on $\mu$.

For a homogeneous space $X$, we denote that $\widehat{X}=X \times(0, \infty)$ the upper halfspace of $X \times \mathbb{R}$, and that $\widehat{B}(x, r)=B(x, r) \times(0, r)$. For a Borel measure $\sigma$ on $X$, we study the operator $T$ defined by

$$
T(f d \sigma)(x, t)=\int_{X} K_{t}(x, y) f(y) d \sigma(y), \quad(x, t) \in \widehat{X},
$$

with the nonnegative kernel $K_{t}(x, y)$ which is lower semi-continuous.

Let $\Omega=\left\{\Omega_{a}: a \in X\right\}$ be a family of nonempty subsets in $\widehat{X}$. For each $a \in X$, set $\Omega_{a}(r)=\left\{x \in X:(x, r) \in \Omega_{a}\right\}$ (the cross-section of $\Omega_{a}$ at height $r$ ). Following $[9]$, for $\alpha>0$ and $(x, r) \in \widehat{X}$, we define

$$
S_{\alpha}(x, r)=\left\{a \in X: \Omega_{a}(r) \cap B(x, \alpha r) \neq \varnothing\right\} .
$$

We suppose that, for each $a \in X, a \in \Omega_{a}(r)$ for all $r>0$, and $\Omega_{a}\left(r^{\prime}\right) \subset \Omega_{a}(r)$ if $r^{\prime} \leq r$. It is clear that, for each $x \in X, S_{\alpha}\left(x, r^{\prime}\right) \subset S_{\alpha}(x, r)$ if $r^{\prime} \leq r$, and $\lim _{r \rightarrow \infty} S_{\alpha}(x, r)=X$.

Theorem 1.1. Let $1 \leq p<q<\infty$ and $0<\alpha<2 \kappa$. Assume that $\sigma$ and $\omega$ are Borel measures on $X$, which satisfy the condition: There exists a positive constant $C_{1}$ such that

$$
\left|S_{\alpha}\left(x, \frac{4 \kappa}{\alpha}(2 r+t)\right)\right|_{\omega}^{1 / q}\left(\int_{X \backslash B(x, r)} K_{t}(x, y)^{p^{\prime}} d \sigma(y)\right)^{1 / p^{\prime}} \leq C_{1},
$$

if $1<p<\infty$, or

$$
\left|S_{\alpha}\left(x, \frac{4 \kappa}{\alpha}(2 r+t)\right)\right|_{\omega}^{1 / q} \operatorname{ess} \sup _{\sigma}\left\{K_{t}(x, y): y \in X \backslash B(x, r)\right\} \leq C_{1},
$$

if $p=1$, for all $(x, t) \in \widehat{X}$ and $r \geq 0$, where ess $\sup _{\sigma}$ denotes the essential supremum with respect to the measure $\sigma$.

Then the weak-type inequality

$$
\left|\left\{a \in X: \sup _{(x, t) \in \Omega_{a}} T(f d \sigma)(x, t)>\lambda\right\}\right|_{\omega}^{1 / q} \leq \frac{C}{\lambda}\left(\int_{X} f(x)^{p} d \sigma(x)\right)^{1 / p}
$$

holds for all $\sigma$-measurable functions $f \geq 0$. 
We shall say that an operator $T$ defined by (1.2) is an operator of potential type if its kernel $K$ satisfies the condition: For a fixed constant $0<\alpha<2 \kappa$, there exists a positive constant $C_{0}$ such that

$K_{t}(x, y) \leq C_{0} K_{t^{\prime}}\left(x^{\prime}, y\right)$ whenever $\left(x^{\prime}, t^{\prime}\right) \in \widehat{B}\left(x, \frac{8 \kappa^{2}}{\alpha}(r+t)\right)$ and $y \in X \backslash B(x, r)$, for all $(x, t) \in \widehat{X}$ and $r \geq 0$. (See also [8] for an essentially equivalent definition.)

Theorem 1.2. Let $1 \leq p<q<\infty$ and $0<\alpha<2 \kappa$, and let $\sigma$ and $\omega$ be locally finite Borel measures on $X$. Assume that the kernel $K$ satisfies condition (1.7); then the weak-type inequality (1.6) holds for all $\sigma$-measurable functions $f \geq 0$ if and only if there exists a positive constant $C_{1}$ such that

$$
\left|S_{\alpha}\left(x, \frac{4 \kappa}{\alpha} t\right)\right|_{\omega}^{1 / q}\left(\int_{X} K_{t}(x, y)^{p^{\prime}} d \sigma(y)\right)^{1 / p^{\prime}} \leq C_{1},
$$

if $1<p<\infty$, or

$$
\left|S_{\alpha}\left(x, \frac{4 \kappa}{\alpha} t\right)\right|_{\omega}^{1 / q} \operatorname{ess} \sup _{\sigma}\left\{K_{t}(x, y): y \in X\right\} \leq C_{1},
$$

if $p=1$, for all $(x, t) \in \widehat{X}$.

\section{Proof of Theorem 1.1}

We shall assume that $1<p<\infty$. For $p=1$, only some mild modifications of the following proof are needed.

For any fixed function $f \geq 0$ and number $\lambda>0$, we set

$$
E_{\lambda}=\left\{a \in X: \sup _{(x, t) \in \Omega_{a}} T(f d \sigma)(x, t)>\lambda\right\} .
$$

Let $B_{R}$ in $X$ be a ball centered at some fixed $x_{0} \in X$ with radius $R>0$. We consider the sets

$$
E_{\lambda}^{*}(R)=\left\{x \in B_{R}: \sup _{0<t \leq R} T(f d \sigma)(x, t)>\lambda\right\},
$$

and

$$
\begin{gathered}
E_{\lambda}(R)=\left\{a \in X: T(f d \sigma)(x, t)>\lambda \text { for some }(x, t) \in \Omega_{a},\right. \\
\text { with } \left.x \in B_{R} \text { and } 0<t \leq d(x)\right\},
\end{gathered}
$$

where

$$
d(x)=\sup \{t \in(0, R]: T(f d \sigma)(x, t)>\lambda\} .
$$

Obviously, we have $E_{\lambda}^{*}(R) \subset E_{\lambda}(R) \subset E_{\lambda}$, and $\lim _{R \rightarrow \infty} E_{\lambda}(R)=E_{\lambda}$.

We first claim that, for each $x \in E_{\lambda}^{*}(R)$ there exists $r(x) \geq(\alpha / 2 \kappa) d(x)$ (we recall that $\alpha<2 \kappa)$ such that

$$
\left|S_{\alpha}\left(x, \frac{4 \kappa}{\alpha} r(x)\right)\right|_{\omega} \leq \frac{C}{\lambda^{q}}\|f\|_{L^{p}(d \sigma)}^{q-p} \int_{B(x, r(x))} f(y)^{p} d \sigma(y),
$$

where the constant $C$ is independent of $x$ and $R$. 
Assuming the last claim for a moment, we finish the proof of the theorem. We may assume that $\sup \left\{r(x): x \in E_{\lambda}^{*}(R)\right\}<\infty$; otherwise (1.6) would follow trivially from

$$
|X|_{\omega}=\sup _{x \in E_{\lambda}^{*}(R)}\left|S_{\alpha}\left(x, \frac{4 \kappa}{\alpha} r(x)\right)\right|_{\omega} \leq \frac{C}{\lambda^{q}}\|f\|_{L^{p}(d \sigma)}^{q-p} \int_{X} f(x)^{p} d \sigma(x) .
$$

Applying the covering lemma for homogeneous spaces (see [2]) with the family of balls $\left\{B(x, r(x)): x \in E_{\lambda}^{*}(R)\right\}$, we obtain a countable, pairwise disjoint subfamily $\left\{B\left(x_{k}, r_{k}\right)\right\}_{k=0}^{\infty}$, where $r_{k}=r\left(x_{k}\right)$, so that $E_{\lambda}^{*}(R) \subset \bigcup_{k} B\left(x_{k}, 4 \kappa r_{k}\right)$, and $r(x) \leq 2 r_{k}$ for all $x \in B\left(x_{k}, 4 \kappa r_{k}\right)$.

We next claim that

$$
E_{\lambda}(R) \subset \bigcup_{k} S_{\alpha}\left(x_{k}, \frac{4 \kappa}{\alpha} r_{k}\right)
$$

Indeed, if $a \in E_{\lambda}(R)$ then there is $(x, t) \in \Omega_{a}$ so that $x \in E_{\lambda}^{*}(R)$ and $t \leq d(x)$. Thus, $x \in B\left(x_{k}, 4 \kappa r_{k}\right)$ and $t \leq d(x) \leq(2 \kappa / \alpha) r(x) \leq(4 \kappa / \alpha) r_{k}$ for some $k$. Therefore, $\left(x,(4 \kappa / \alpha) r_{k}\right) \in \Omega_{a}$ by our assumption on $\Omega$. This implies that

$$
x \in \Omega_{a}\left(\frac{4 \kappa}{\alpha} r_{k}\right) \cap B\left(x_{k}, \alpha \frac{4 \kappa}{\alpha} r_{k}\right),
$$

and therefore, $a \in S_{\alpha}\left(x_{k},(4 \kappa / \alpha) r_{k}\right)$.

By virtue of (2.6) and (2.5), we have

$$
\begin{aligned}
\left|E_{\lambda}(R)\right|_{\omega} & \leq \sum_{k}\left|S_{\alpha}\left(x_{k}, \frac{4 \kappa}{\alpha} r_{k}\right)\right|_{\omega} \\
& \leq \frac{C}{\lambda^{q}}\|f\|_{L^{p}(d \sigma)}^{q-p} \sum_{k} \int_{B\left(x_{k}, r_{k}\right)} f(x)^{p} d \sigma(x) \\
& \leq \frac{C}{\lambda^{q}}\|f\|_{L^{p}(d \sigma)}^{q-p} \int_{X} f(x)^{p} d \sigma(x) \\
& \leq \frac{C}{\lambda^{q}}\left(\int_{X} f(x)^{p} d \sigma(x)\right)^{q / p}
\end{aligned}
$$

where we have used the fact that $\left\{B\left(x_{k}, r_{k}\right)\right\}$ is pairwise disjoint. By letting $R \rightarrow$ $\infty$, we obtain (1.6).

We now prove (2.5). If $x \in E_{\lambda}^{*}(R)$, then there exists $t$ with $(\alpha / 2 \kappa) d(x) \leq$ $t \leq d(x)$ so that $T(f d \sigma)(x, t)>\lambda$. To simplify the notation, we shall denote $S(r)=S_{\alpha}(x,(4 \kappa / \alpha) r)$. Without loss of generality, we can assume that there is $r>0$ so that

$$
\frac{C_{1}}{|S(2 r+t)|_{\omega}^{1 / q}}\left(\int_{X}\left(\frac{2 f(y)}{\lambda}\right)^{p} d \sigma(y)\right)^{1 / p}<1 .
$$

Otherwise, (1.6) would follow trivially from

$$
|X|_{\omega}^{1 / q}=\lim _{r \rightarrow \infty}|S(2 r+t)|_{\omega}^{1 / q} \leq \frac{2 C_{1}}{\lambda}\left(\int_{X} f(y)^{p} d \sigma(y)\right)^{1 / p}
$$


Let $d_{0}$ be the greatest lower bound for the $r$ satisfying (2.7); then, by using Hölder's inequality and condition (1.4), we have

$$
\begin{aligned}
& \int_{X \backslash B(x, r)} K_{t}(x, y) f(y) d \sigma(y)=\frac{\lambda}{2} \int_{X \backslash B(x, r)} K_{t}(x, y) \frac{2 f(y)}{\lambda} d \sigma(y) \\
\leq & \frac{\lambda}{2}\left(\int_{X \backslash B(x, r)} K_{t}(x, y)^{p^{\prime}} d \sigma(y)\right)^{1 / p^{\prime}}\left(\int_{X \backslash B(x, r)}\left(\frac{2 f(y)}{\lambda}\right)^{p} d \sigma(y)\right)^{1 / p} \\
\leq & \frac{C_{1} \lambda}{2|S(2 r+t)|_{\omega}^{1 / q}}\left(\int_{X}\left(\frac{2 f(y)}{\lambda}\right)^{p} d \sigma(y)\right)^{1 / p} \leq \frac{\lambda}{2}
\end{aligned}
$$

for all $r>d_{0}$. Therefore, we obtain either

$$
\int_{B(x, r)} K_{t}(x, y) f(y) d \sigma(y)>\frac{\lambda}{2}, \quad \text { if } r>d_{0}>0,
$$

or

$$
K_{t}(x, x) f(x)|\{x\}|_{\sigma}>\frac{\lambda}{2}, \quad \text { if } d_{0}=0 .
$$

If $d_{0}=0$ then

$$
\begin{aligned}
\frac{\lambda}{2} & <K_{t}(x, x) f(x)|\{x\}|_{\sigma} \leq \int_{B(x, t)} K_{t}(x, y) f(y) d \sigma(y) \\
& \leq\left(\int_{X} K_{t}(x, y)^{p^{\prime}} d \sigma(y)\right)^{1 / p^{\prime}}\left(\int_{B(x, t)} f(y)^{p} d \sigma(y)\right)^{1 / p} \\
& \leq \frac{C_{1}}{|S(t)|_{\omega}^{1 / q}}\left(\int_{B(x, t)} f(y)^{p} d \sigma(y)\right)^{1 / p}
\end{aligned}
$$

and so that

$$
\begin{aligned}
|S(t)|_{\omega} & \leq\left(\frac{2 C_{1}}{\lambda}\right)^{q}\left(\int_{B(x, t)} f(y)^{p} d \sigma(y)\right)^{q / p} \\
& \leq \frac{C}{\lambda^{q}}\|f\|_{L^{p}(d \sigma)}^{q-p} \int_{B(x, t)} f(y)^{p} d \sigma(y) .
\end{aligned}
$$

If $d_{0}>0$ then there is $r_{0}>0$ such that

$$
\frac{C_{1}}{\left|S\left(2 r_{0}+t\right)\right|_{\omega}^{1 / q}}\left(\int_{X}\left(\frac{2 f(y)}{\lambda}\right)^{p} d \sigma(y)\right)^{1 / p}<1,
$$

and

$$
\frac{C_{1}}{\left|S\left(r_{0}+t\right)\right|_{\omega}^{1 / q}}\left(\int_{X}\left(\frac{2 f(y)}{\lambda}\right)^{p} d \sigma(y)\right)^{1 / p} \geq 1 .
$$

We now choose a (finite or infinite) decreasing sequence $\left\{r_{j}\right\}_{j=1}^{n}$ of positive numbers such that

$$
\left|S\left(r_{j}+t\right)\right|_{\omega} \leq 2^{-j}\left|S\left(r_{0}+t\right)\right|_{\omega} \leq\left|S\left(2 r_{j}+t\right)\right|_{\omega}
$$


for $j=1,2, \ldots, n$. Due to $p<q$, we have

$$
\sum_{j=1}^{n}\left(\frac{\left|S\left(r_{j}+t\right)\right|_{\omega}}{\left|S\left(r_{0}+t\right)\right|_{\omega}}\right)^{1 / p-1 / q} \leq \sum_{j=1}^{\infty}\left(\frac{1}{2^{j}}\right)^{1 / p-1 / q}=\beta<\infty
$$

Thus, we obtain from (2.8) that

$$
\frac{\lambda}{2 \beta} \sum_{j=1}^{n}\left(\frac{\left|S\left(r_{j}+t\right)\right|_{\omega}}{\left|S\left(r_{0}+t\right)\right|_{\omega}}\right)^{1 / p-1 / q} \leq \sum_{j=1}^{n} \int_{B_{j} \backslash B_{j+1}} K_{t}(x, y) f(y) d \sigma(y)
$$

where $B_{j}=B\left(x, r_{j}\right)$, and $B_{n+1}=\{x\}$ if $n$ is finite. Hence, there exists $j_{0}$ such that

$$
\begin{aligned}
& \frac{\lambda}{2 \beta}\left(\frac{\left|S\left(r_{j_{0}}+t\right)\right|_{\omega}}{\left|S\left(r_{0}+t\right)\right|_{\omega}}\right)^{1 / p-1 / q}<\int_{B_{j_{0}} \backslash B_{j_{0}+1}} K_{t}(x, y) f(y) d \sigma(y) \\
\leq & \left(\int_{B_{j_{0}} \backslash B_{j_{0}+1}} K_{t}(x, y)^{p^{\prime}} d \sigma(y)\right)^{1 / p^{\prime}}\left(\int_{B_{j_{0} \backslash B_{j_{0}+1}}} f(y)^{p} d \sigma(y)\right)^{1 / p} \\
\leq & \frac{C_{1}}{\left|S\left(2 r_{j_{0}+1}+t\right)\right|_{\omega}^{1 / q}}\left(\int_{B_{j_{0}}} f(y)^{p} d \sigma(y)\right)^{1 / p} \\
\leq & \frac{2^{1 / q} C_{1}}{\left|S\left(r_{j_{0}}+t\right)\right|_{\omega}^{1 / q}}\left(\int_{B_{j_{0}}} f(y)^{p} d \sigma(y)\right)^{1 / p}
\end{aligned}
$$

the last step follows from (2.12). Consequently, by using (2.11), we get

$$
\begin{aligned}
\left|S\left(r_{j_{0}}+t\right)\right|_{\omega} & \leq \frac{C}{\lambda^{p}}\left|S\left(r_{0}+t\right)\right|_{\omega}^{1-p / q} \int_{B\left(x, r_{j_{0}}\right)} f(y)^{p} d \sigma(y) \\
& \leq \frac{C}{\lambda^{q}}\|f\|_{L^{p}(d \sigma)}^{q-p} \int_{B\left(x, r_{j_{0}}\right)} f(y)^{p} d \sigma(y) \\
& \leq \frac{C}{\lambda^{q}}\|f\|_{L^{p}(d \sigma)}^{q-p} \int_{B\left(x, r_{j_{0}}+t\right)} f(y)^{p} d \sigma(y) .
\end{aligned}
$$

This completes the proof of (2.5), if we choose $r(x)=r_{j_{0}}+t$ and note $t \geq$ $(\alpha / 2 \kappa) d(x)$. Therefore Theorem 1.1 is proved.

\section{Proof of Theorem 1.2}

We first show that conditions (1.8) and (1.9) imply conditions (1.4) and (1.5), respectively, if the kernel $K$ satisfies condition (1.7). Then the sufficient part of the theorem follows from Theorem 1.1. To see this, we fix $x \in X, t>0$ and $r \geq 0$. Then, $(x, 2 r+t) \in \widehat{B}\left(x,\left(8 \kappa^{2} / \alpha\right)(r+t)\right)$, since $0<\alpha<2 \kappa$, and so that

$$
K_{t}(x, y) \leq C_{0} K_{2 r+t}(x, y)
$$


for $y \in X \backslash B(x, r)$, by (1.7). Therefore

$$
\begin{aligned}
& \left|S_{\alpha}\left(x, \frac{4 \kappa}{\alpha}(2 r+t)\right)\right|_{\omega}^{1 / q}\left(\int_{X \backslash B(x, r)} K_{t}(x, y)^{p^{\prime}} d \sigma(y)\right)^{1 / p^{\prime}} \\
\leq & \left|S_{\alpha}\left(x, \frac{4 \kappa}{\alpha}(2 r+t)\right)\right|_{\omega}^{1 / q}\left(\int_{X \backslash B(x, r)} K_{2 r+t}(x, y)^{p^{\prime}} d \sigma(y)\right)^{1 / p^{\prime}} \\
\leq & \left|S_{\alpha}\left(x, \frac{4 \kappa}{\alpha} t\right)\right|_{\omega}^{1 / q}\left(\int_{X} K_{t}(x, y)^{p^{\prime}} d \sigma(y)\right)^{1 / p^{\prime}} \leq C_{1} .
\end{aligned}
$$

The proof for the case $p=1$ is similar.

We now prove the necessity part of the theorem. We consider the case of $1<$ $p<\infty$ first. Let $\left(x_{0}, t_{0}\right) \in \widehat{X}$ be temporarily fixed, and for each positive integer $N$, define

$$
K_{t_{0}}^{N}\left(x_{0}, y\right)= \begin{cases}K_{t_{0}}\left(x_{0}, y\right) & \text { if } K\left(x_{0}, y\right)<N, \\ N & \text { if } K_{t_{0}}\left(x_{0}, y\right) \geq N .\end{cases}
$$

Let $R>0$ be arbitrary fixed constant and $0<\beta<1 / C_{0}$, we now take

$$
f(y)=K_{t_{0}}^{N}\left(x_{0}, y\right)^{p^{\prime} / p} \chi_{B\left(x_{0}, R\right)}(y)
$$

and $\lambda=\beta \int_{B\left(x_{0}, R\right)} K_{t_{0}}^{N}\left(x_{0}, y\right)^{p^{\prime}} d \sigma(y)$. We note that $\lambda<\infty$ since $\sigma$ is locally finite. If $a \in S_{\alpha}\left(x_{0},(4 \kappa / \alpha) t_{0}\right)$, then there is $x \in \Omega_{a}\left((4 \kappa / \alpha) t_{0}\right) \cap B\left(x_{0}, 4 \kappa t_{0}\right)$, and hence $\left(x,(4 \kappa / \alpha) t_{0}\right) \in \Omega_{a} \cap \widehat{B}\left(x_{0},\left(8 \kappa^{2} / \alpha\right) t_{0}\right)$, since $0<\alpha<2 \kappa$. Apply condition (1.7) with $r=0$, we get $K_{t_{0}}\left(x_{0}, y\right) \leq C_{0} K_{(4 \kappa / \alpha) t_{0}}(x, y)$ for all $y \in X$, and therefore, $K_{t_{0}}^{N}\left(x_{0}, y\right) \leq C_{0} K_{(4 \kappa / \alpha) t_{0}}(x, y)$ for all $y \in X$. Thus

$$
T(f d \sigma)\left(x, \frac{4 \kappa}{\alpha} t_{0}\right) \geq \frac{1}{C_{0}} \int_{B\left(x_{0}, R\right)} K_{t_{0}}^{N}\left(x_{0}, y\right) K_{t_{0}}^{N}\left(x_{0}, y\right)^{p^{\prime} / p} d \sigma(y)>\lambda,
$$

which implies that

$$
S_{\alpha}\left(x_{0}, \frac{4 \kappa}{\alpha} t_{0}\right) \subset\left\{a \in X: \sup _{(x, t) \in \Omega_{a}} T(f d \sigma)(x, t)>\lambda\right\} .
$$

Therefore, by using the weak-type inequality (1.6), we obtain

$$
\begin{aligned}
& \beta\left|S_{\alpha}\left(x_{0}, \frac{4 \kappa}{\alpha} t_{0}\right)\right|_{\omega}^{1 / q} \int_{B\left(x_{0}, R\right)} K_{t_{0}}^{N}\left(x_{0}, y\right)^{p^{\prime}} d \sigma(y) \\
\leq & \lambda\left|\left\{a \in X: \sup _{(x, t) \in \Omega_{a}} T(f d \sigma)(x, t)>\lambda\right\}\right|_{\omega}^{1 / q} \\
\leq & C\left(\int_{B\left(x_{0}, R\right)} K_{t_{0}}^{N}\left(x_{0}, y\right)^{p^{\prime}} d \sigma(y)\right)^{1 / p} .
\end{aligned}
$$

Thus, (1.8) follows by division, and then letting $R \rightarrow \infty$ and $N \rightarrow \infty$.

For the case $p=1$, we fix $\left(x_{0}, t_{0}\right) \in \widehat{X}$ temporarily, and for each positive integer $N>4 \kappa t_{0}$ set

$$
\Lambda_{N}=\min \left\{N, \operatorname{ess} \sup _{\sigma}\left\{K_{t_{0}}\left(x_{0}, y\right): y \in X\right\}\right\}
$$


We could assume that $\Lambda_{N}>0$, otherwise (1.9) would hold trivially. Let $R$ be an arbitrary positive number, and choose a number $0<\eta<1$ so that the set

$$
U_{R, N}=\left\{y \in B\left(x_{0}, N\right): K_{t_{0}}\left(x_{0}, y\right) \geq \eta \Lambda_{N}\right\}
$$

has nonzero and finite $\sigma$-measure, since $\sigma$ is locally finite. Now, take $f=\chi_{U_{R, N}}$ and $\lambda=\beta \Lambda_{N}\left|U_{R, N}\right|_{\sigma}$ with $0<\beta<\eta / C_{0}$. As before, if $a \in S_{\alpha}\left(x_{0},(4 \kappa / \alpha) t_{0}\right)$, then there is $x \in \Omega_{a}\left((4 \kappa / \alpha) t_{0}\right) \cap B\left(x_{0}, 4 \kappa t_{0}\right)$ and hence condition (1.7) implies that

$$
T(f d \sigma)\left(x, \frac{4 \kappa}{\alpha} t_{0}\right) \geq \frac{1}{C_{0}} \int_{X} K_{t_{0}}\left(x_{0}, y\right) \chi_{U_{R, N}}(y) d \sigma(y) \geq \frac{\eta}{C_{0}} \Lambda_{N}\left|U_{R, N}\right|_{\sigma}>\lambda,
$$

since $N>4 \kappa t_{0}$. Therefore, by the weak-type inequality (1.6), we obtain

$$
\begin{aligned}
& \beta\left|S_{\alpha}\left(x_{0}, \frac{4 \kappa}{\alpha} t_{0}\right)\right|_{\omega}^{1 / q} \Lambda\left|U_{R, N}\right|_{\sigma} \\
\leq & \lambda\left|\left\{a \in X: \sup _{(x, t) \in \Omega_{a}} T(f d \sigma)(x, t)>\lambda\right\}\right|_{\omega}^{1 / q} \\
\leq & C\left(\int_{X} \chi_{U_{R, N}}(y) d \sigma(y)\right)=C\left|U_{R, N}\right|_{\sigma} .
\end{aligned}
$$

Again, (1.9) follows by division, and then letting $R \rightarrow \infty$ and $N \rightarrow \infty$. This completes the proof of Theorem 1.2.

\section{REFERENCES}

[1] P. Ahern and A. Nagel, Strong $L^{p}$ estimates for maximal functions with respect to singular measures; with applications to exceptional sets, Duke Math. J. 53 (1986), 359-393. MR 88m: 42037

[2] R. Coifman and G. Weiss, Extensions of Hardy spaces and their use in analysis, Bull. Amer. Math. Soc. 83 (1977), 569-645. MR 56:6264

[3] I. Genebashvili, A. Gogatishvili, and V. Kokilashvili, Criteria of general weak type inequalities for integral transforms with positive kernels, Proc. Georgian Acad. Sci. (Math.) 1 (1993), 11-34. MR 94j:42030

[4] R. Macias and C. Segovia, Lipschitz functions on spaces of homogeneous type, Adv. in Math. 33 (1979), 257-270. MR 81c:32017a

[5] B. A. Mair and D. Singman, A generalized Fatou theorem, Trans. Amer. Math. Soc. 300 (1987), 705-719. MR 88f:31011

[6] A. Nagel and E. M. Stein, On certain maximal functions and approach regions, Adv. in Math. 54 (1984), 83-106. MR 86a:42026

[7] W. Pan, Weighted norm inequalities for certain maxmial operators with approach regions, Harmonic Analysis (M.-T. Cheng et al., ed.), Lecture Notes in Math., vol. 1494, SpringerVerlag, Berlin and Heidelberg, 1991, pp. 167-175. MR 94a:42015

[8] E. T. Sawyer and R. L. Wheeden, Weighted inequalities for fractional integrals on Euclidean and homogeneous spaces, Amer. J. Math. 114 (1992), 813-874. MR 94i:42024

[9] J. Sueiro, On maximal functions and Poisson-Szegö intergrals, Trans. Amer. Math. Soc. 298 (1986), 653-669. MR 87m:42017

Department of Mathematics and Computer Science, University of Missouri-St. Louis, St. Louis, Missouri 63121

E-mail address: zhao@greatwall.cs.umsl.edu 